\title{
Polarimetric SAR Speckle Noise Model
}

\author{
Carlos López-Martínez, Student Member, IEEE, and Xavier Fàbregas, Member, IEEE
}

\begin{abstract}
Synthetic aperture radar (SAR) data are affected by speckle noise, originated by the SAR system's coherent nature. The problem of speckle noise in one-dimensional (1-D) data is already solved, as speckle has a multiplicative characteristic. SAR polarimetry represents an extension to multidimensional data by the use of polarization wave diversity. As a consequence of the existence of a correlation degree between the SAR images, the 1-D speckle noise model cannot be extended to multidimensional SAR data. This paper is devoted to present a completely new speckle noise model for the complex covariance matrix describing polarimetric SAR data in the distributed scatterers case. As will be shown, this new model is able to identify which are the noise mechanisms in all the covariance matrix elements. The speckle noise model is validated by using real $L$-band polarimetric data acquired with the German E-SAR sensor.
\end{abstract}

Index Terms-Covariance matrix, noise modeling, speckle noise, synthetic aperture radar (SAR) polarimetry.

\section{INTRODUCTION}

$\mathbf{S}_{\text {tent }}$ YNTHETIC aperture radar (SAR) is a well-established technology for remote sensing applications. As it has been extensively reported in the literature, the information that these type of systems can gather has a crucial importance for monitoring natural features and changes on the earth's surface. SAR systems have first been employed in a single-channel configuration to obtain high spatial resolution information about the reflectivity properties of the imaged scene [1]. The availability of multidimensional SAR systems has made possible to increase the amount of available information about the earth's surface. In particular, SAR polarimetry (PolSAR) is a well-established multidimensional SAR technique based on acquiring earth's surface information by means of using a pair of orthogonal polarizations for the transmitted and received electromagnetic fields [2]. Nowadays, there exists an extensive amount of techniques based on PolSAR data, e.g., terrain classification, surface parameter estimation, biomass estimation, etc. The importance of PolSAR can be also seen from the sensor's point of view, as airborne PolSAR systems already exist, and satellite systems are planned.

Speckle noise [3], [4] is one of the most important problems of SAR data. In order to obtain high spatial resolution in the azimuth dimension, SAR systems coherently record the returned echoes. The speckle noise is precisely originating from this coherent nature. Speckle noise in single-channel SAR systems is

\footnotetext{
Manuscript received September 29, 2002; revised May 20, 2003. This work was supported by the Departament Universitats Recerca i Societat de la Informació (DURSI) from the Catalonian Government and by the Spanish CICYT project TIC2002-04451-C02-01.

The authors are with the Signal Theory and Communications Department, Technical University of Catalonia (UPC), E-08034 Barcelona, Spain.

Digital Object Identifier 10.1109/TGRS.2003.815240
}

a solved problem, as it is well known that it has a multiplicative nature for the amplitude and an additive nature for the phase [3], [5], [6]. On the contrary, the speckle noise problem for PolSAR data is still not solved. An extension of the single-channel model is not possible, as the SAR images present generally a correlation degree between them. Hence, the speckle noise nature for PolSAR data is still not known [7], [8]. SAR data modeling and SAR data filtering (i.e., information estimation) are two different aspects of the same problem, as the availability of a polarimetric noise model would make a correct signal estimation possible.

Several alternatives have been investigated in order to reduce speckle noise in multidimensional SAR data, with special interest in PolSAR data. These techniques can be divided into two big groups depending on which is the final purpose. The first group embraces all those techniques assuming multidimensional SAR data as a type of diversity, combining all the channels to derive speckle-free images [9]-[11]. These approaches are characterized for keeping the spatial resolution but loosing all the polarimetric information. The second group contains all those techniques based on obtaining the speckle-free input images, therefore maintaining the correlation information between them [8], [12]. This second group of techniques is basically based on an spatial processing of the SAR images, therefore, affecting the spatial resolution. Relating the two groups of approaches, it is clear that there is a trade-off between spatial resolution maintenance and the preservation of the interchannel information. This compromise is due, in part, to the fact that the information about the speckle noise nature for PolSAR data is not complete.

In this paper, the authors present a complete speckle noise model for single-look PolSAR data. This new model is able to identify the speckle noise characteristics for all the covariance matrix elements. As shown, the developed noise model is also in accordance with all the previous theory about speckle. The model will be qualitatively and quantitatively validated with real PolSAR data.

The present paper has been divided as follows. Section II contains a brief introduction to PolSAR, focused on data description. Section III is devoted to present the speckle noise model theory, together with an analysis of its properties. A qualitative and quantitative validation is reported in Section IV. Finally, Section V presents the main conclusions derived from the study presented in this paper.

\section{Polarimetric SAR DATA DESCRIPTION}

A polarimetric SAR sensor measures the $2 \times 2$ complex scattering matrix $[S]$, for each resolution cell, which relates the components of the scattered electromagnetic field with the 
illuminating field, for a particular polarization basis. For the linear polarization basis case

$$
[S]=\left[\begin{array}{ll}
S_{\mathrm{hh}} & S_{\mathrm{hv}} \\
S_{\mathrm{vh}} & S_{\mathrm{vv}}
\end{array}\right]
$$

where $h$ and $v$ represent the horizontal and vertical linear polarizations, respectively. $S_{p q}$ is the scattering coefficient relating the illuminating field with $q$-polarization and the received field in $p$-polarization. $[S]$ can be decomposed in an orthogonal matrix basis, yielding to the target vector's concept [13]. For the lexicographic decomposition basis, the target vector $\mathbf{k}$ is

$$
\mathbf{k}=\left[\begin{array}{llll}
S_{\mathrm{hh}} & S_{\mathrm{hv}} & S_{\mathrm{vh}} & S_{\mathrm{vv}}
\end{array}\right]^{T}
$$

where ${ }^{T}$ indicates transpose. For the backscattering direction, due to the reciprocity theorem under the BSA convention [2], i.e., $S_{\mathrm{hv}}=S_{\mathrm{vh}}$, k can be simplified to

$$
\mathbf{k}=\left[\begin{array}{lll}
S_{\mathrm{hh}} & \sqrt{2} S_{\mathrm{hv}} & S_{\mathrm{vv}}
\end{array}\right]^{T}
$$

where $\sqrt{2}$ is introduced to maintain the vector's norm $|\mathbf{k}|^{2}$ or span. $[S]$ characterizes completely the scattering process for deterministic scatterers. On the contrary, it fails to characterize the scattering process for distributed scatterers [14], i.e., random targets. For this type of scatterers, as a consequence of the random changes from pixel to pixel, the matrix $[S]$ is, therefore, random. Based on the SAR's coherent nature, under the Gaussian scatterer assumption, $\mathbf{k}$ can be modeled by a multivariate, complex, zero-mean, Gaussian probability density function (pdf) $\mathcal{N}(\mathbf{0},[\mathcal{C}])[15],[16]$

$$
p_{\mathbf{k}}(\mathbf{k})=\frac{1}{\pi^{3}|[C]|} \exp \left(-\mathbf{k}^{\dagger}|[C]|^{-1} \mathbf{k}\right)
$$

where $\dagger$ represents the transpose complex conjugate of a vector, and $|[C]|$ denotes the determinant of $[C]$. This pdf is completely determined by the $3 \times 3$ complex, Hermitian, covariance matrix $[C]$, defined as

$$
\begin{aligned}
{[C] } & =E\left\{\mathbf{k k}^{\dagger}\right\} \\
& =\left[\begin{array}{ccc}
E\left\{\left|S_{\mathrm{hh}}\right|^{2}\right\} & \sqrt{2} E\left\{S_{\mathrm{hh}} S_{\mathrm{hv}}^{*}\right\} & E\left\{S_{\mathrm{hh}} S_{\mathrm{vv}}^{*}\right\} \\
\sqrt{2} E\left\{S_{\mathrm{hv}} S_{\mathrm{hh}}^{*}\right\} & 2 E\left\{\left|S_{\mathrm{hv}}\right|^{2}\right\} & \sqrt{2} E\left\{S_{\mathrm{hv}} S_{\mathrm{vv}}^{*}\right\} \\
E\left\{S_{\mathrm{vv}} S_{\mathrm{hh}}^{*}\right\} & \sqrt{2} E\left\{S_{\mathrm{vv}} S_{\mathrm{vh}}^{*}\right\} & E\left\{\left|S_{\mathrm{vv}}\right|^{2}\right\}
\end{array}\right]
\end{aligned}
$$

where $E\{\cdot\}$ represents the ensemble average, and * is the complex conjugate of a complex quantity. Most of natural scenes are considered as distributed scatterers; therefore, they are completely determined, in polarimetric terms, by $[C]$ and not by $[S]$. For distributed scatterers, $[S]$ has five independent parameters, whereas $[C]$ has nine. This difference comes from the fact that $[C]$ contains information concerning the data's correlation structure.
Assuming statistical ergodicity and homogeneity, $[C]$ can be estimated substituting the ensemble average by spatial averaging, known as multilook

$$
[Z]=\frac{1}{n} \sum_{l=1}^{n}[C]_{l}
$$

where $n$ represents the number of averaged pixels, and $[C]_{l}$ is the covariance matrix of a particular pixel defined as $[C]_{l}=$ $\mathbf{k}_{l} \mathbf{k}_{l}^{\dagger}$. As the sample covariance matrix $[Z]$ is derived from $[S]$, it is affected by speckle noise in such a way that the larger the number of averaged pixels, the lower the speckle noise content [13], [17]. In this case, speckle reduction (i.e., signal estimation) is obtained at the expense of spatial resolution. The statistics of the covariance matrix $[Z]$ have been found to be the Wishart pdf $\mathcal{W}([\mathcal{C}], n)[15],[16],[18]$

$$
p_{[Z]}([Z])=\frac{n^{q n}|[Z]|^{n-q} \exp \left(-n \operatorname{tr}\left([C]^{-1}[Z]\right)\right)}{K(n, q)|[C]|^{n}}
$$

where $K(n, q)$ is

$$
K(n, q)=\pi^{(1 / 2) q(q-1)} \Gamma(n) \cdots \Gamma(n-q+1) .
$$

$\operatorname{tr}(\cdot)$ is the matrix trace, and $q$ represents the number of channels. In the case of a monostatic PolSAR system configuration, $q$ equals 3 .

From the pdf given by (7), it is possible to derive the pdf corresponding to all the quantities within the covariance matrix. See [15] and [16] for a complete and detailed statistical analysis of the Wishart pdf, as well as the pdfs of the associated parameters. These pdfs will be employed to derive the speckle noise model for the covariance matrix.

\section{POlARIMETRIC SAR DATA SPECKLE NOISE MODEL}

All the covariance matrix $[C]$ elements are obtained as the product of two elements of the scattering matrix $[S]$. Therefore, in order to derive a noise model for $[C]$, only a particular element will be studied. Any element of the $[C]$ matrix can be written as the complex Hermitian product $S_{\mathrm{pq}} S_{\mathrm{rs}}^{*}$, where $p, q, r$, and $s$ belong to a pair of orthogonal polarization states. The effects of using a different polarization basis will be commented on later in this paper.

As mentioned previously, $S_{\mathrm{pq}} S_{\mathrm{rs}}^{*}$ has a random nature due to speckle. Its statistical behavior is determined by the complex correlation coefficient

$$
\rho=\frac{E\left\{S_{\mathrm{pq}} S_{\mathrm{rs}}^{*}\right\}}{\sqrt{E\left\{\left|S_{\mathrm{pq}}\right|^{2}\right\} E\left\{\left|S_{\mathrm{rs}}\right|^{2}\right\}}}=|\rho| \exp \left(j \phi_{x}\right)
$$

where $|\rho|$ is called coherence, and $\phi_{x}$ is known as the effective phase difference. The average power in the two channels is defined as $\psi=\left(E\left\{\left|S_{\mathrm{pq}}\right|^{2}\right\} E\left\{\left|S_{\mathrm{rs}}\right|^{2}\right\}\right)^{1 / 2}$. The terms $S_{\mathrm{pq}} S_{\mathrm{rs}}^{*}$ can be written as

$$
S_{\mathrm{pq}} S_{\mathrm{rs}}^{*}=\left|S_{\mathrm{pq}} S_{\mathrm{rs}}^{*}\right| \exp \left(j\left(\phi_{\mathrm{pq}}-\phi_{\mathrm{rs}}\right)\right)=z \exp (j \phi)
$$

where $z$ is the amplitude, and $\phi$ represents the measured phase difference. In order to derive a speckle model for (10), first, a 
noise model will be derived for the phase difference $\phi$ in the complex plane, i.e., $\exp (j \phi)$. Then, the amplitude information $z$ will be included to derive the final speckle noise model.

\section{A. Phase Difference Noise Model}

Based on the Gaussian scattering model for distributed scatterers, the expression for the measured phase difference $\phi$ pdf, obtained from the Wishart distribution (7), is [15], [16]

$$
\begin{aligned}
p_{\phi}(\phi)=\frac{\Gamma\left(n+\frac{1}{2}\right)\left(1-|\rho|^{2}\right)^{n} \beta}{2 \sqrt{\pi} \Gamma(n)\left(1-\beta^{2}\right)^{n+1 / 2}} & \\
& +\frac{\left(1-|\rho|^{2}\right)^{n}}{2 \pi}{ }_{2} F_{1}\left(n, 1 ; \frac{1}{2} ; \beta^{2}\right)
\end{aligned}
$$

where $\beta=|\rho| \cos \left(\phi-\phi_{x}\right)$, and ${ }_{2} F_{1}(a, b ; c ; z)$ represents the Gauss hypergeometric function. Based on (11), the phase difference $\phi$ follows an additive noise model in the real plane [19]

$$
\phi=\phi_{x}+v
$$

where $\phi_{x}$ is the original phase difference without noise, and $v$ represents a zero-mean noise depending on $|\rho|$ and $n$, and independent from $\phi_{x}$. The measured phase can be coded in the complex unitary circle as a unit amplitude phasor

$$
\exp (j \phi)=\cos (\phi)+j \sin (\phi) .
$$

In this case, only the real part of (13) will be analyzed, since the imaginary part phase noise model can be derived in the same way. Based on the additive phase noise model (12), the real part of (13) can be decomposed as

$$
\cos (\phi)=\cos \left(\phi_{x}+v\right)=\cos \left(\phi_{x}\right) \cos (v)-\sin \left(\phi_{x}\right) \sin (v) .
$$

In (14), the phase term $\phi_{x}$ has been separated from the noise contribution $v$. The terms $\cos (v)$ and $\sin (v)$ are only due to the noise term $v$. In [20], the authors gave the expression for the distributions of $\cos (v)$ and $\sin (v)$. These two noise contributions can be represented as the addition of their mean values plus random terms with the same variances [21]

$$
\begin{aligned}
& v_{1}=\cos (v)=N_{c}+v_{1}^{\prime} \\
& v_{2}=\sin (v)=N_{s}+v_{2}^{\prime}
\end{aligned}
$$

where $N_{s}=0$. On the contrary, $N_{c}$, which represents the mean value of $\cos (v)$, equals, for $n=1$ (i.e., one-look imagery)

$$
N_{c}=\frac{\pi}{4}|\rho|_{2} F_{1}\left(\frac{1}{2}, \frac{1}{2} ; 2 ;|\rho|^{2}\right) .
$$

As it will be shown later, this is the key parameter to define the speckle noise model for (10). Fig. 1 shows the behavior of $N_{c}$ as a function of $|\rho|$. As (17) shows, this parameter has the same information content as $|\rho|$. Therefore, instead of taking it as a noise parameter, it will be considered as a signal term. Using (14)-(16), the real and imaginary parts of (13) can be written as

$$
\operatorname{Re}\{\exp (j \phi)\}=N_{c} \cos \left(\phi_{x}\right)+v_{1}^{\prime} \cos \left(\phi_{x}\right)-v_{2}^{\prime} \sin \left(\phi_{x}\right)
$$

$\operatorname{Im}\{\exp (j \phi)\}=N_{c} \sin \left(\phi_{x}\right)+v_{1}^{\prime} \sin \left(\phi_{x}\right)+v_{2}^{\prime} \cos \left(\phi_{x}\right)$.

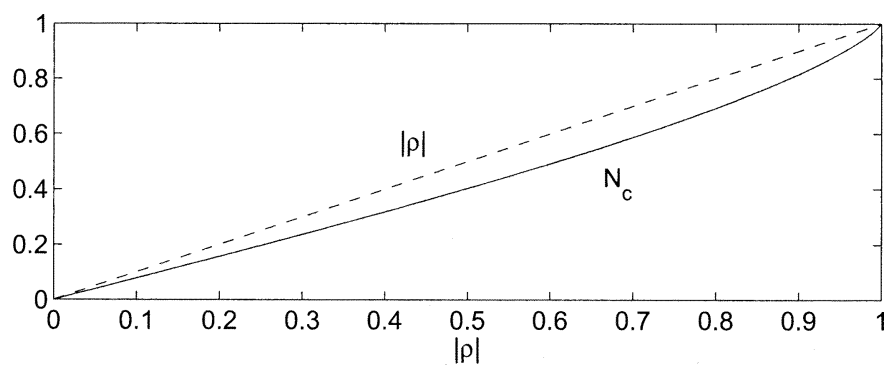

Fig. 1. Comparison between $N_{c}$ and $|\rho|$.

$\operatorname{Re}\{\cdot\}$ and $\operatorname{Im}\{\cdot\}$ denote the real and imaginary parts, respectively. The parameters $N_{c}, v_{1}^{\prime}$, and $v_{2}^{\prime}$ are due to the phase noise term $v$. The noise model for the complex phase term $\exp (j \phi),(18)$ and (19), has been validated for interferometric SAR (InSAR) data as shown in [20]. Under the assumption of a Gaussian scatterer, as PolSAR data and InSAR data are distributed as $\mathcal{W}([C], \mathrm{n})$ [15], it can be considered that the phase difference noise model (12) is also valid for PolSAR data [22], [23].

\section{B. Hermitian Product Noise Model}

In this section, the noise model given by (18) and (19) is taken as a starting point to obtain the complete noise model for the covariance matrix terms $S_{\mathrm{pq}} S_{\mathrm{rs}}^{*}$. Hence, the amplitude information $z$ has to be introduced within (18) and (19)

$\operatorname{Re}\{z \exp (j \phi)\}=N_{c} z \cos \left(\phi_{x}\right)+z v_{1}^{\prime} \cos \left(\phi_{x}\right)-z v_{2}^{\prime} \sin \left(\phi_{x}\right)$

$\operatorname{Im}\{z \exp (j \phi)\}=N_{c} z \sin \left(\phi_{x}\right)+z v_{1}^{\prime} \sin \left(\phi_{x}\right)+z v_{2}^{\prime} \cos \left(\phi_{x}\right)$.

In order to derive the speckle noise model for $S_{\mathrm{pq}} S_{\mathrm{rs}}^{*}$, homogeneous data will be assumed. In the following, the three terms of (20) and (21) will be studied in detail to identify noise sources, i.e., sources of randomness, and therefore, to see how they degrade the useful information content, i.e., the signal's mean value. The model is derived on the analysis of the real and imaginary parts of the Hermitian product, despite a complex model will be presented.

1) Noise Model: The first term is $N_{c} z \cos \left(\phi_{x}\right)$ for $\operatorname{Re}\left\{S_{\mathrm{pq}} S_{\mathrm{rs}}^{*}\right\}$ and $N_{c} z \sin \left(\phi_{x}\right)$ for $\operatorname{Im}\left\{S_{\mathrm{pq}} S_{\mathrm{rs}}^{*}\right\}$. For homogeneous data, the terms $N_{c}$, as well as $\cos \left(\phi_{x}\right)$ and $\sin \left(\phi_{x}\right)$ are constant values. Hence, the first term in (20) and (21) is completely determined statistically by the amplitude information $z$. The mean and the variance of this term, for the real part case (20) based on the expressions given in [16], are

$$
\begin{aligned}
E\left\{N_{c} z \cos \left(\phi_{x}\right)\right\}= & N_{c} \cos \left(\phi_{x}\right) E\{z\} \\
= & \psi N_{c} \cos \left(\phi_{x}\right) \frac{\pi}{4}{ }_{2} F_{1}\left(-\frac{1}{2},-\frac{1}{2} ; 1 ;|\rho|^{2}\right) \\
\operatorname{var}\left\{N_{c} z \cos \left(\phi_{x}\right)\right\}= & \psi^{2} N_{c}^{2} \cos ^{2}\left(\phi_{x}\right) \\
& \cdot\left(1+|\rho|^{2}-\left(\frac{\pi}{4}\right)^{2}\right. \\
& \left.\cdot{ }_{2} F_{1}^{2}\left(-\frac{1}{2},-\frac{1}{2} ; 1 ;|\rho|^{2}\right)\right)
\end{aligned}
$$

where $\operatorname{var}\{\cdot\}$ denotes the variance. 


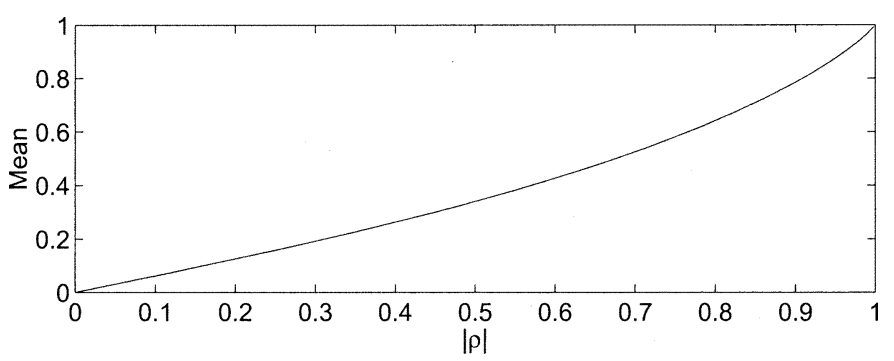

Fig. 2. Mean value of the first speckle noise term.

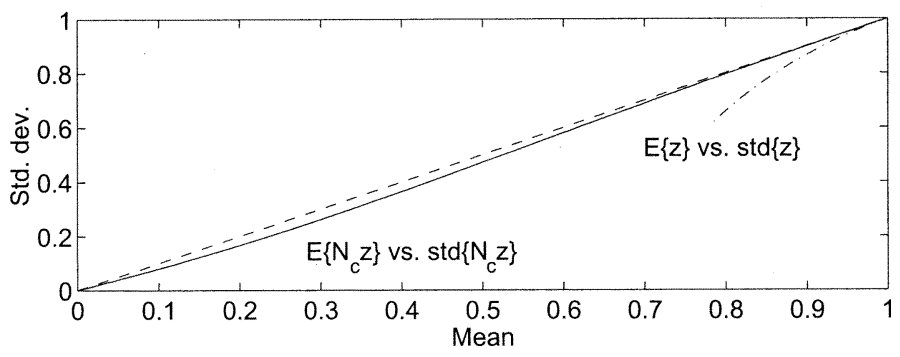

Fig. 3. Mean value versus standard deviation of the first speckle noise term. Dashed-dotted line shows the relation without introducing $N_{c}$, whereas the continuous line presents the relation introducing $N_{c}$.

Fig. 2 depicts the mean value given by (22), assuming $\psi=1$ and $\cos \left(\phi_{x}\right)=1$. Fig. 3 shows the effect of the parameter $N_{c}$ over the mean and the variance of the first term of (20), also considering $\psi=1$ and $\cos \left(\phi_{x}\right)=1$. If the parameter $N_{c}$ is not taken into consideration, the mean and the variance given by (22) and (23) correspond to the statistical parameters of the amplitude $z$. In this case, if the standard deviation is plotted as a function of the mean, a clear relation cannot be established, as shown by the dashed-dotted line in Fig. 3. On the other hand, if $N_{c}$ is introduced, and the standard deviation is plotted as a function of the mean, it can be observed from the continuous line in Fig. 3 that the relation between them is very close to an equality relation, i.e., $\operatorname{std}\left\{N_{c} z \cos \left(\phi_{x}\right)\right\} \simeq$ $\operatorname{abs}\left(E\left\{N_{c} z \cos \left(\phi_{x}\right)\right\}\right)$, where $\operatorname{std}\{\cdot\}$ is the standard deviation. As a consequence, the term $N_{c} z \cos \left(\phi_{x}\right)$ can be approximated by a multiplicative noise term in the following way:

$$
N_{c} z \cos \left(\phi_{x}\right) \simeq N_{c} \bar{z} \cos \left(\phi_{x}\right) n_{m}=\psi N_{c} \cos \left(\phi_{x}\right) \bar{z}_{n} n_{m}
$$

where $n_{m}$ denotes an homogeneous noise term with $E\left\{n_{m}\right\}=$ 1 and $\operatorname{var}\left\{n_{m}\right\}=1$. The parameter $\bar{z}$ is the mean amplitude and $\bar{z}_{n}$ is the same quantity but normalized, i.e., $\bar{z}=\psi \bar{z}_{n}$. For the imaginary part case (21), the term $\cos \left(\phi_{x}\right)$ has to be substituted by $\sin \left(\phi_{x}\right)$ in (22)-(24).

The second term in (20) and (21) is $z v_{1}^{\prime} \cos \left(\phi_{x}\right)$ and $z v_{1}^{\prime} \sin \left(\phi_{x}\right)$ for the real and imaginary parts, respectively. As

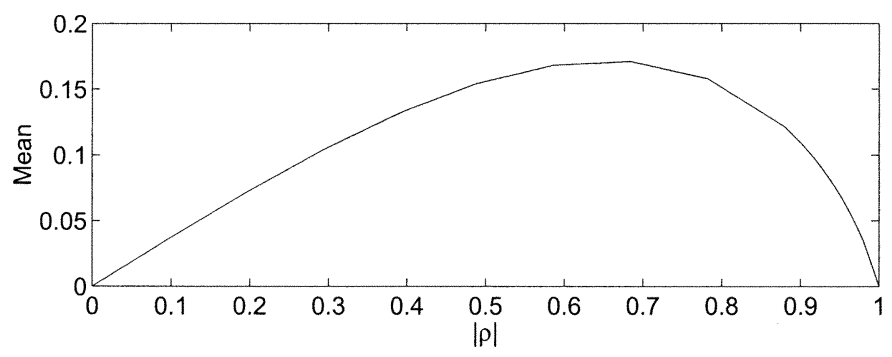

Fig. 4. Mean value of the second speckle noise term.

in the previous case, assuming homogeneous data, $\cos \left(\phi_{x}\right)$ and $\sin \left(\phi_{x}\right)$ are constants. Thus, this second term is statistically determined by $z v_{1}^{\prime}$. From (15), $v_{1}^{\prime}=\cos (v)-N_{c}$, and on the basis of the expressions given in [16], the mean value of $z v_{1}^{\prime} \cos \left(\phi_{x}\right)$ is

$$
\begin{aligned}
E\left\{z v_{1}^{\prime} \cos \left(\phi_{x}\right)\right\}= & \cos \left(\phi_{x}\right)\left(E\{z \cos (v)\}-N_{c} E\{z\}\right) \\
= & \psi \cos \left(\phi_{x}\right)\left(|\rho|-N_{c} \frac{\pi}{4}\right. \\
& \left.\cdot{ }_{2} F_{1}\left(-\frac{1}{2},-\frac{1}{2} ; 1 ;|\rho|^{2}\right)\right) .
\end{aligned}
$$

Assuming $\psi=1$ and $\cos \left(\phi_{x}\right)=1$, the maximum value of (25) is lower than 0.2 (see Fig. 4). In order to obtain the value of the variance for the second term (15) is used again. The variance value is as shown in (26) at the bottom of the page. As (26) shows, the variance of the second term presents a complicated expression, making difficult to extract conclusions concerning its dependence with the different parameters. For this reason, an approximation for (26) has been considered. The type of curve used to approximate (26) has been selected taking into account its dependence on $|\rho|$ (see Fig. 5), but also its similar dependence on $|\rho|$ compared to the third term variance of (20), as it will be shown in (31). The approximation is hence

$$
\operatorname{var}\left\{z v_{1}^{\prime} \cos \left(\phi_{x}\right)\right\} \simeq \frac{1}{2} \psi^{2} \cos ^{2}\left(\phi_{x}\right)\left(1-|\rho|^{2}\right)^{1.64} .
$$

As it can be seen from Fig. 5, for $\psi=1$ and $\cos \left(\phi_{x}\right)=1$, the approximation presents a very small error with respect to the real value. Besides, it can be seen that the variance (26) has a well-defined dependence on the coherence $|\rho|$.

In a similar way as it has been done with the first term of (20) and (21), in this case, the random variable defined by $z v_{1}^{\prime} \cos \left(\phi_{x}\right)$ can be divided into its mean value plus a random term with the same variance

$$
z v_{1}^{\prime} \cos \left(\phi_{x}\right)=\psi \cos \left(\phi_{x}\right)\left\{\left(|\rho|-N_{c} \bar{z}_{n}\right)+n_{a 1}\right\}
$$

$$
\begin{aligned}
\operatorname{var}\left\{z v_{1}^{\prime} \cos \left(\phi_{x}\right)\right\}= & \cos ^{2}\left(\phi_{x}\right)\left(E\left\{\left(z v_{1}^{\prime}-\overline{z v_{1}^{\prime}}\right)^{2}\right\}\right)=\cos ^{2}\left(\phi_{x}\right)\left(E\left\{z^{2}\left(\cos (v)-N_{c}\right)^{2}\right\}-E^{2}\left\{z v_{1}^{\prime}\right\}\right) \\
=\psi^{2} \cos ^{2}\left(\phi_{x}\right) & {\left[\frac{1}{2}\left(1-|\rho|^{2}\right)+2|\rho|^{2}+N_{c}^{2}+N_{c}^{2}|\rho|^{2}-N_{c}|\rho| \frac{9 \pi}{8}{ }_{2} F_{1}\left(-\frac{1}{2},-\frac{1}{2} ; 2 ;|\rho|^{2}\right)\right.} \\
& \left.-\left(|\rho|-N_{c} \frac{\pi}{4}{ }_{2} F_{1}\left(-\frac{1}{2},-\frac{1}{2} ; 1 ;|\rho|^{2}\right)\right)^{2}\right]
\end{aligned}
$$




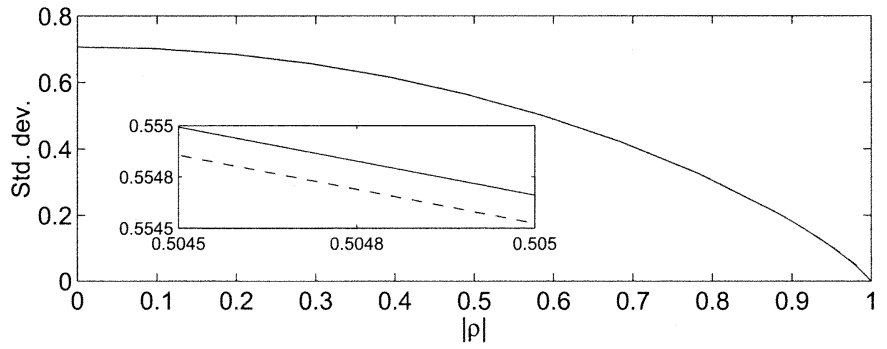

Fig. 5. Standard deviation of the second speckle noise term. The dashed line represents the approximation, and the solid line is the exact value.

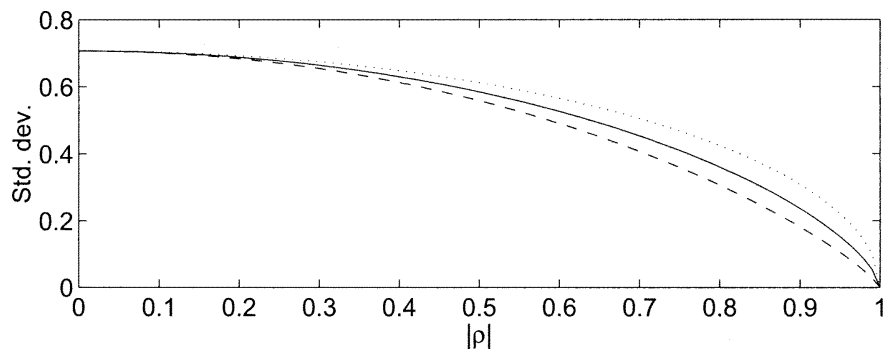

Fig. 6. Standard deviation corresponding to the additive noise terms. Dashed line: $\operatorname{std}\left\{n_{a 1}\right\}$. Dotted line: $\operatorname{std}\left\{n_{a 2}\right\}$. Continuous line: Approximation for $\operatorname{std}\left\{n_{\text {ar }}\right\}$ and $\operatorname{std}\left\{n_{\text {ai }}\right\}$.

where $n_{a 1}$ denotes a noise term with $E\left\{n_{a 1}\right\}=0$ and a variance

$$
\operatorname{var}\left\{n_{a 1}\right\}=\frac{\operatorname{var}\left\{z v_{1}^{\prime}\right\}}{\psi^{2}} .
$$

In this case, the value of $\psi$ is extracted from the mean and variance values, as the final noise model will be easily interpreted.

The last term of (20) and (21), $z v_{2}^{\prime} \sin \left(\phi_{x}\right)$ and $z v_{2}^{\prime} \cos \left(\phi_{x}\right)$ respectively, is analyzed next. As it will be seen, it is very similar to the second term. Based on (16) and [16], the mean value is

$$
E\left\{z v_{2}^{\prime} \sin \left(\phi_{x}\right)\right\}=\sin \left(\phi_{x}\right) E\{z \sin (v)\}=0 .
$$

The variance of this third term can be derived also using (15)

$$
\begin{aligned}
\operatorname{var}\left\{z v_{2}^{\prime} \sin \left(\phi_{x}\right)\right\} & =E\left\{\sin ^{2}\left(\phi_{x}\right)\left(z v_{2}^{\prime}-\overline{z v_{2}^{\prime}}\right)^{2}\right\} \\
& =\frac{1}{2} \psi^{2} \sin ^{2}\left(\phi_{x}\right)\left(1-|\rho|^{2}\right) .
\end{aligned}
$$

Comparing (31) and (27), as shown by Fig. 6, the variance of this term has the same type of behavior as the second term (26): the lower the coherence value $|\rho|$, the larger the variance value. Hence, it can be deduced that the last two terms within (20) and (21) will determine the speckle behavior for low coherences. As performed before, this third term can be written as

$$
z v_{2}^{\prime} \sin \left(\phi_{x}\right)=\psi \sin \left(\phi_{x}\right) n_{a 2}
$$

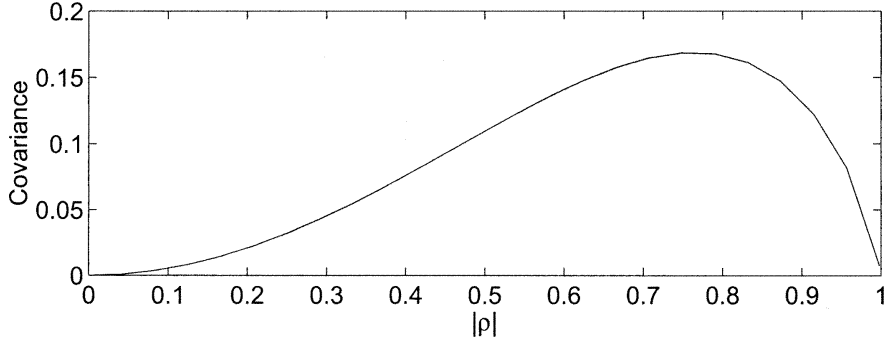

Fig. 7. Covariance value between the first and the second speckle noise terms.

where $n_{a 2}$ determines a random term with $E\left\{n_{a 2}\right\}=0$ and a variance

$$
\operatorname{var}\left\{n_{a 2}\right\}=\frac{\operatorname{var}\left\{z v_{2}^{\prime}\right\}}{\psi^{2}} .
$$

The above analysis has shown which is the behavior of the individual terms of (20) and (21). The first term can be thought of as a multiplicative noise term, (24), whereas the last two terms (28) and (32) can be interpreted as additive noise terms. In order to obtain a final noise model, it is necessary to determine the correlations between the three terms of (20) and (21). First of all, the covariance between the first an the second term, for the real part case, is obtained as in (34), shown at the bottom of the page.

Fig. 7 presents the plot of this covariance value. The covariance between the first and the third term of (20) is

$$
\begin{aligned}
& E\left\{\cos \left(\phi_{x}\right)\left(N_{c} z-\overline{N_{c} z}\right) \sin \left(\phi_{x}\right)\left(z v_{2}^{\prime}\right)\right\} \\
& =\cos \left(\phi_{x}\right) \sin \left(\phi_{x}\right)\left(N_{c} E\left\{z^{2} \sin (v)\right\}-\overline{N_{c} z} E\{z \sin (v)\}\right) \\
& =0 .
\end{aligned}
$$

Equally, the covariance between the second and the third additive terms is zero

$$
\begin{aligned}
& E\left\{\cos \left(\phi_{x}\right)\left(z v_{1}^{\prime}-\overline{z v_{1}^{\prime}}\right) \sin \left(\phi_{x}\right)\left(z v_{2}^{\prime}\right)\right\} \\
& =\cos \left(\phi_{x}\right) \sin \left(\phi_{x}\right)\left(E\left\{z^{2} \cos (v) \sin (v)\right\}-E\left\{z^{2} \sin (v)\right\}\right) \\
& =0
\end{aligned}
$$

The noise model for the elements of the covariance matrix $[C]$ (10) is finally obtained substituting (24), (28) and (32), together with the equivalent expression for the imaginary part, within (20) and (21). These expressions are combined to form the complex Hermitian product speckle noise model

$$
\begin{aligned}
S_{\mathrm{pq}} S_{\mathrm{rs}}^{*}= & \psi N_{c} \bar{z}_{n} n_{m} \exp \left(j \phi_{x}\right) \\
& +\psi\left[\left(|\rho|-N_{c} \bar{z}_{n}\right)+n_{a 1}\right] \exp \left(j \phi_{x}\right) \\
& +\psi n_{a 2} \exp \left(j\left(\phi_{x}+\pi\right)\right) .
\end{aligned}
$$

2) Noise Model Simplification: The approximation introduced within (20) and (21) by (24), has allowed to define a linear noise model for (10) as shown by (37). Three noise

$$
\begin{aligned}
E\left\{\cos \left(\phi_{x}\right)\left(N_{c} z-\overline{N_{c} z}\right) \cos \left(\phi_{x}\right)\left(z v_{1}^{\prime}-\overline{z v_{1}^{\prime}}\right)\right\}=\cos ^{2}\left(\phi_{x}\right)\left(N_{c} E\left\{z^{2} v_{1}^{\prime}\right\}-\overline{N_{c} z \overline{z v_{1}^{\prime}}}\right)=\psi^{2} N_{c} \cos ^{2}\left(\phi_{x}\right) \\
\cdot\left[|\rho| \frac{9 \pi}{16}{ }_{2} F_{1}\left(-\frac{1}{2},-\frac{1}{2} ; 2 ;|\rho|^{2}\right)-N_{c}\left(1+|\rho|^{2}\right)-\frac{\pi}{4}{ }_{2} F_{1}\left(-\frac{1}{2},-\frac{1}{2} ; 1 ;|\rho|^{2}\right)\left(|\rho|-N_{c} \frac{\pi}{4}{ }_{2} F_{1}\left(-\frac{1}{2},-\frac{1}{2} ; 1 ;|\rho|^{2}\right)\right)\right]
\end{aligned}
$$


sources have been identified: a multiplicative noise source given by $n_{m}$ and two additive noise sources defined by $n_{a 1}$ and $n_{a 2}$. As (36) shows, the additive noise components are uncorrelated, making it possible to join them

$$
\begin{aligned}
& \psi n_{\mathrm{ar}}=\psi \cos \left(\phi_{x}\right) n_{a 1}-\psi \sin \left(\phi_{x}\right) n_{a 2} \\
& \psi n_{\mathrm{ai}}=\psi \sin \left(\phi_{x}\right) n_{a 1}+\psi \cos \left(\phi_{x}\right) n_{a 2}
\end{aligned}
$$

where $n_{\text {ar }}$ denotes a single additive term for the complex Hermitian product real part noise model, and $n_{\mathrm{ai}}$ is the additive term for the imaginary part. As $E\left\{n_{a 1}\right\}=0$ and $E\left\{n_{a 2}\right\}=0$, hence, $E\left\{n_{\mathrm{ar}}\right\}=0$ and $E\left\{n_{\mathrm{ai}}\right\}=0$. In order to calculate the variance, as $n_{a 1}$ and $n_{a 2}$ are uncorrelated and $\cos ^{2}\left(\phi_{x}\right)+$ $\sin ^{2}\left(\phi_{x}\right)=1$, the following inequality will be employed

$$
\operatorname{var}\left\{n_{a 1}\right\} \leq \underset{\operatorname{var}\left\{n_{\mathrm{ar}}\right\}}{\operatorname{var}\}} \leq \operatorname{var}\left\{n_{a 2}\right\}
$$

Due to (40), and the similarity between $\operatorname{var}\left\{n_{a 1}\right\}$ and $\operatorname{var}\left\{n_{a 2}\right\}$ (see Fig. 6), the variances of $n_{\text {ar }}$ and $n_{\text {ai }}$ can be approximated by any value between $\operatorname{var}\left\{n_{a 1}\right\}$ and $\operatorname{var}\left\{n_{a 2}\right\}$. For this approximation, a curve removing the dependence on the phase $\phi_{x}$, at the expense of introducing an small error in the final variance, will be taken. In order to minimize this error on average, a mean curve is chosen. The justification for this approximation lies in the fact that a simple noise model can be derived. The selected curve is

$$
\operatorname{var}\left\{n_{\mathrm{ar}}\right\}=\operatorname{var}\left\{n_{\mathrm{ai}}\right\} \simeq \frac{1}{2} \psi^{2}(1-|\rho|)^{1.32}
$$

The effect of this approximation can also be observed in Fig. 6 . Introducing the approximations given by (38) and (39) within (37), the final expression for the speckle noise model for $S_{\mathrm{pq}} S_{\mathrm{rs}}^{*}$ is

$$
\begin{aligned}
S_{\mathrm{pq}} S_{\mathrm{rs}}^{*}= & \psi N_{c} \bar{z}_{n} n_{m} \exp \left(j \phi_{x}\right) \\
& +\psi\left(|\rho|-N_{c} \bar{z}_{n}\right) \exp \left(j \phi_{x}\right)+\psi\left(n_{\mathrm{ar}}+j n_{\mathrm{ai}}\right) .
\end{aligned}
$$

In (42), it can easily be identified that the speckle noise, for any element of the covariance matrix $[C]$, has two different noise components. The first term, given by $\psi N_{c} \bar{z}_{n} n_{m} \exp \left(j \phi_{x}\right)$, has a multiplicative noise characteristic, given by the term $n_{m}$. As this noise term affects equally the real and the imaginary parts of the complex Hermitian product, it only introduces noise in the amplitude, whereas the phase is not altered. The third term, denoted by $\psi\left(n_{\mathrm{ar}}+j n_{\mathrm{ai}}\right)$, introduces noise both in the amplitude and the phase, as the processes $n_{\mathrm{ar}}$ and $n_{\mathrm{ai}}$ are different. The second term $\psi\left(|\rho|-N_{c} \bar{z}_{n}\right) \exp \left(j \phi_{x}\right)$ is just a mean value without noise. Fig. 8 presents a vectorial view of the noise model. As it can be seen, the two first terms only affect the amplitude, whereas the third term defines a cloud which introduces noise, as mentioned, in amplitude and in phase. In the following, the first term of (42) is referred as the multiplicative speckle noise term, whereas the addition of the last two terms will be referred as the additive speckle noise term.

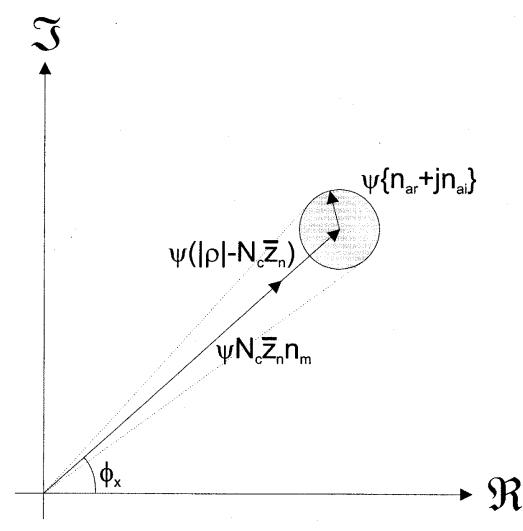

Fig. 8. Speckle noise model scheme.

\section{Speckle Noise Model Interpretation}

Equation (42) has been derived for a general term of the sample covariance matrix given by $S_{\mathrm{pq}} S_{\mathrm{rs}}^{*}$. A diagonal element of the covariance matrix is a particular case, which has the expression $S_{\mathrm{pq}} S_{\mathrm{pq}}^{*}$. Therefore, it can be analyzed as the Hermitian product of two terms $S_{\mathrm{pq}}$ and $S_{\mathrm{pq}}^{*}$ respectively, characterized by a complex correlation coefficient with unit amplitude and a zero phase. In this case, (42) simplifies to

$$
S_{\mathrm{pq}} S_{\mathrm{pq}}^{*}=\psi n_{m} .
$$

The noise terms $n_{\mathrm{ar}}$ and $n_{\mathrm{ai}}$ disappear, as their mean and variance values are zero. As a result, (43) defines the multiplicative speckle noise model for the diagonal terms of the covariance matrix [8]. In the same way, the defined speckle noise model is in accordance with the additive noise model for the phase difference in the real plane [19] by construction. Therefore, the derived speckle noise model is in accordance with the previous theory about speckle noise, generalizing it for all the covariance matrix elements. That is, (42) defines which is the speckle noise nature for the diagonal, as well as for the off-diagonal covariance matrix elements.

As presented previously, the multiplicative speckle noise term $n_{m}$ is homogeneous, as it does not depend either on the power term $\psi$ or on the complex correlation coefficient $\rho$. On the other hand, the additive noise terms $n_{\mathrm{ar}}$ and $n_{\mathrm{ai}}$ cannot be considered as homogeneous, since their variances depend on $|\rho|$ [see (41)]. Despite these dependencies, the final nature of the complex Hermitian product speckle noise, i.e., the combination of the multiplicative and the additive speckle noise terms, is clearly determined by the complex correlation coefficient $\rho$. As observed in (42), the first additive term, i.e., $\psi N_{c} \bar{z}_{n} n_{m} \exp \left(j \phi_{x}\right)$, contains the complex coefficient $N_{c} \exp \left(j \phi_{x}\right)$. This coefficient determines the influence of the multiplicative term $n_{m}$ on the final speckle noise. For low coherences, since $N_{c}$ is low and the variances of the additive noise terms $n_{\text {ar }}$ and $n_{\text {ai }}$ present maximum values, speckle noise is dominated by an additive behavior. When $|\rho|=0$, speckle noise is completely additive as $N_{c}=0$. The situation is more complex when the coherence has a medium or a high value, since in this case, the phase $\phi_{x}$ also determines how the multiplicative and the additive noise terms are combined. The higher the coherence, the lower the additive noise variances, but at the same time, the higher the contribution 
of the multiplicative noise term as $N_{c}$ grows with $|\rho|$. Regardless of the phase $\phi_{x}$, it can be concluded that the higher the coherence, the lower the contribution of the additive noise term and the higher the importance of the multiplicative term. When $|\rho|$ is one, speckle noise is completely multiplicative. Using the same argument, the real and imaginary parts of the first additive term of (42) depend, respectively, on $\cos \left(\phi_{x}\right)$ and $\sin \left(\phi_{x}\right)$. Hence, whenever any of these terms equals zero, it will produce the corresponding real or imaginary part of the complex Hermitian product to contain only the additive speckle noise term. Therefore, this can produce the additive speckle noise to be more important than the multiplicative one, even for high coherences. In conclusion, despite the multiplicative term is homogeneous and the additive noise terms depends only on $|\rho|$, the final nature of speckle noise depends on $\rho$, as it controls the contribution of the multiplicative speckle noise term.

In order to see the behavior of each of the noise terms in (42), it is necessary to find a way to separate those components. As it has been mentioned, the term containing the multiplicative noise term is completely characterized, in statistical terms, by the amplitude $z$. Therefore, this term can be obtained multiplying the amplitude $z$ by the term $N_{c} \exp \left(j \phi_{x}\right) . N_{c} \exp \left(j \phi_{x}\right)$ can be derived from the complex correlation coefficient $\rho$, transforming its amplitude to $N_{c}$ by using (24). The term $\psi\left\{\left(|\rho|-N_{c} \bar{z}_{n}\right)\right\} \exp \left(j \phi_{x}\right)+\psi\left(n_{\mathrm{ar}}+j n_{\mathrm{ai}}\right)$ can be obtained, thus, by subtracting $N_{c} z \exp \left(j \phi_{x}\right)$ from $S_{\mathrm{pq}} S_{\mathrm{rs}}^{*}$.

Equation (42) represents the speckle noise model for a particular element of the sample covariance matrix $[Z]$ in the case of single-look SAR imagery. Since all the elements of this matrix are obtained as the Hermitian product of a pair of SAR images, it is straightforward to derive a noise model for $[Z]$, since (42) is valid for all its elements. Additionally, since the Wishart pdf is completely characterized by $[C]$, it is not necessary to investigate speckle noise in higher moments, since these can be obtained as a function of [C] [24].

Some polarimetric data processing techniques are based on changing the polarization basis [25]. The basis change can be interpreted as a matrix rotation, whose main effect is a change on the data's correlation structure, varying the correlation coefficients between the different channels [13]. Therefore, a polarimetric basis change also alters the speckle noise characteristics of the covariance matrix elements.

\section{SPECKLE Noise Model VALIDATION}

The rest of this paper is focused on validating the proposed speckle noise model using real PolSAR data. These data correspond to an L-band, fully polarimetric dataset acquired over the Oberpfaffenhofen test site, located nearby the German city of Munich, with the airborne E-SAR system, operated by DLR. These polarimetric data are referred to the horizontal $h$ and vertical $v$ linear polarization basis. Fig. 9 presents the complex correlation coefficient of the term $S_{\mathrm{hh}} S_{\mathrm{vv}}^{*}$. The speckle noise model is tested by using scatter diagrams in which the standard deviation is plotted against the mean employing $7 \times 7$ pixel nonoverlapping windows. Fig. 10 depicts the scatter diagram for the term $\operatorname{Re}\left\{S_{\mathrm{hh}} S_{\mathrm{vv}}^{*}\right\}$. As pointed out in [8], this diagram confirms that speckle noise is neither multiplicative nor additive.

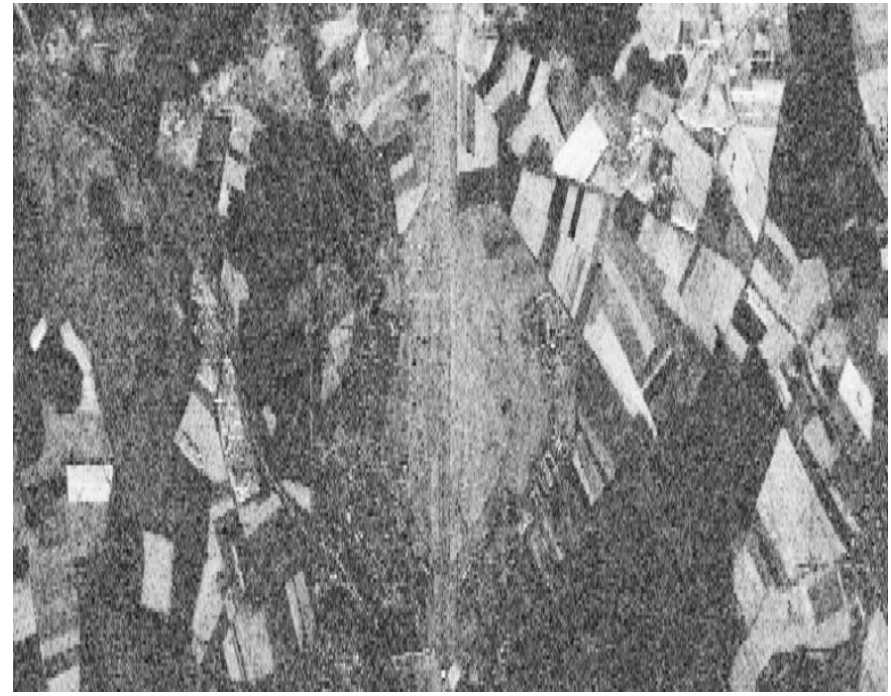

(a)

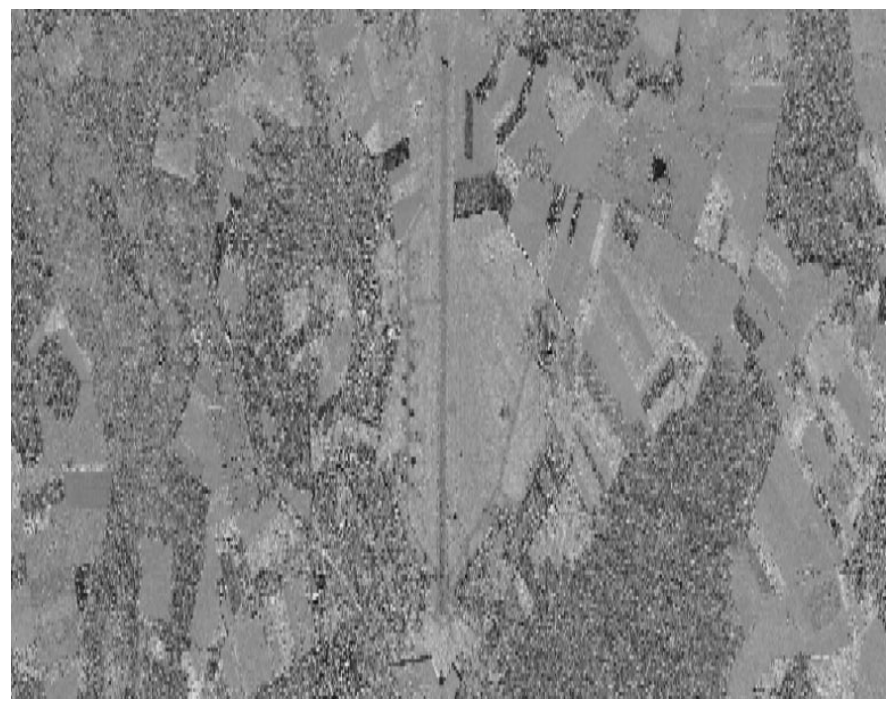

(b)

Fig. 9. L-band PolSAR data. Pictures present the complex correlation coefficient for the term $S_{\mathrm{hh}} S_{\mathrm{vv}}^{*}$. (a) Amplitude or coherence. (b) Phase.

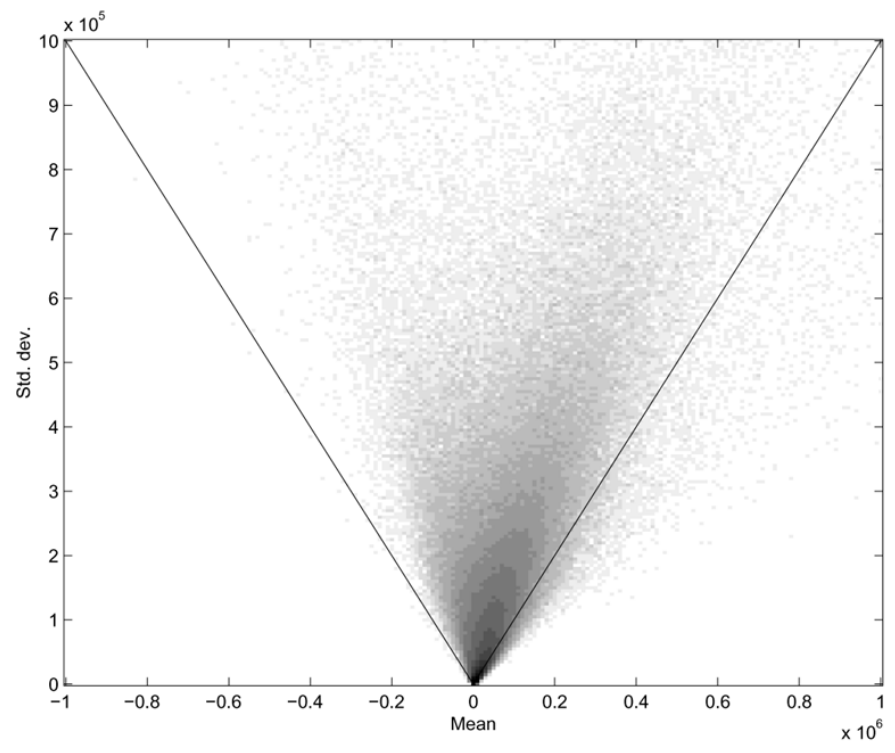

Fig. 10. Scatter diagram for $\operatorname{Re}\left\{S_{\mathrm{hh}} S_{\mathrm{vv}}^{*}\right\}$. 
Total
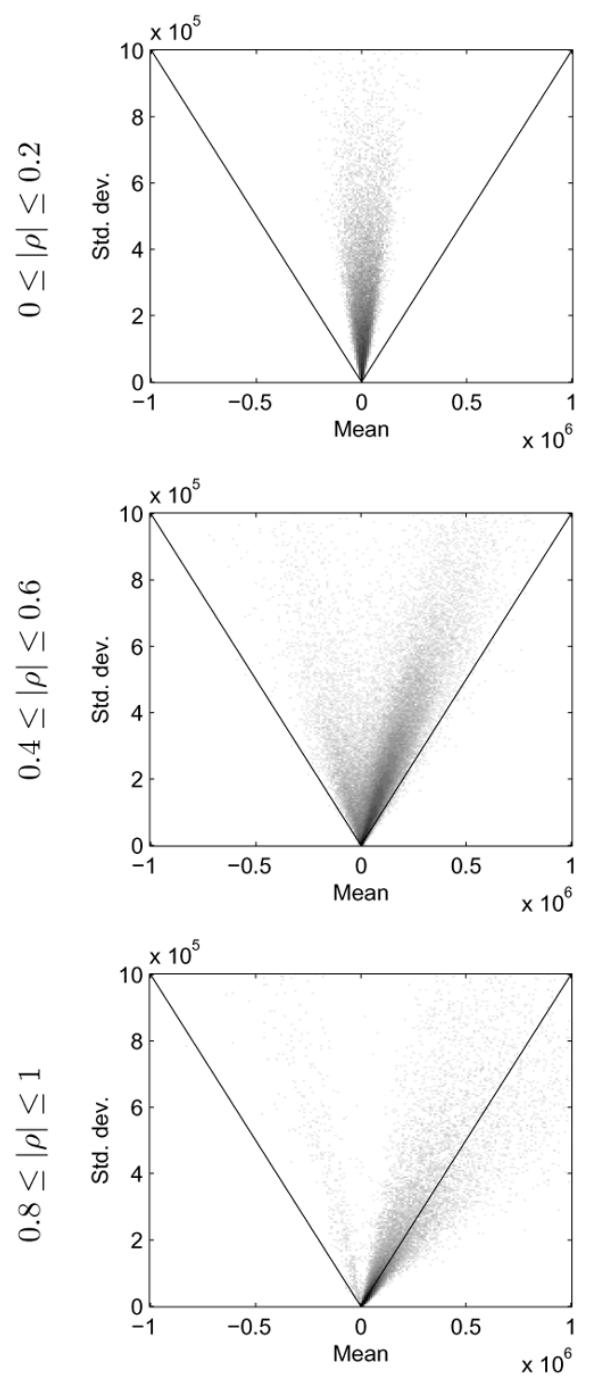

Mult. noise term
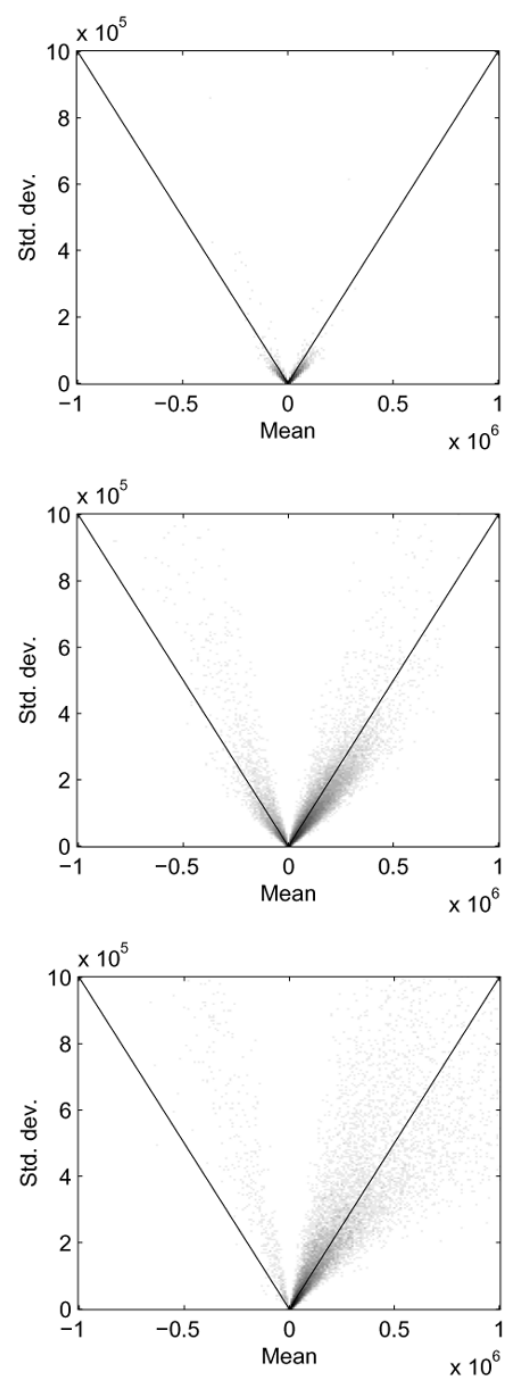

Add. noise term
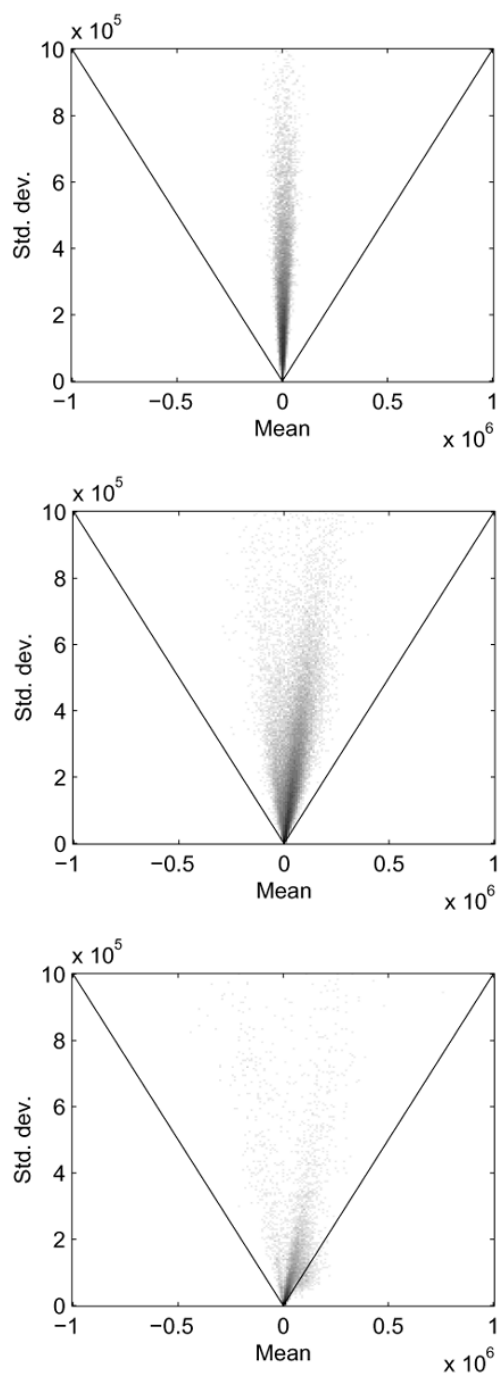

Fig. 11. Mean versus standard deviation scatter diagrams for different coherence ranges and the different terms of the speckle noise model for Re $\left\{S_{\mathrm{hh}} S_{\mathrm{vv}}^{*}\right\}$. The first column represents $\operatorname{Re}\left\{S_{\mathrm{hh}} S_{\mathrm{vv}}^{*}\right\}$, and the second column gives the multiplicative speckle noise term, whereas the third column corresponds to the additive speckle noise term.

First of all, the speckle noise dependence on the coherence $|\rho|$ is tested. In this case, the phase dependence is not considered, as it will be analyzed later in this section. Therefore, only $\operatorname{Re}\left\{S_{\mathrm{hh}} S_{\mathrm{vv}}^{*}\right\}$ is taken into consideration. The term $\operatorname{Im}\left\{S_{\mathrm{hh}} S_{\mathrm{vv}}^{*}\right\}$, as it can be concluded from (42), presents the same type of behavior, with the only difference that it depends on $\sin \left(\phi_{x}\right)$ instead of $\cos \left(\phi_{x}\right)$. Data are divided into three coherence ranges: from 0 to 0.2 (low coherence), from 0.4 to 0.6 (medium coherence), and from 0.8 to 1 (high coherence). For every case, the scatter diagrams corresponding to $\operatorname{Re}\left\{S_{\mathrm{hh}} S_{\mathrm{vv}}^{*}\right\}$, its multiplicative term, and its additive term are calculated. Such an analysis is presented in Fig. 11. For low coherences, the scatter diagram of $\operatorname{Re}\left\{S_{\mathrm{hh}} S_{\mathrm{vv}}^{*}\right\}$ is very similar to the one corresponding to the additive speckle noise term, i.e., a signal with a zero mean and a variance depending on $\psi$. The multiplicative term is negligible in terms of mean and standard deviation with respect to the additive speckle noise term. For a medium coherence, it can be observed that there is not a dominant noise mechanism. Finally, for the case of a high coherence, the similarity of the scatter diagram of $\operatorname{Re}\left\{S_{\mathrm{hh}} S_{\mathrm{vv}}^{*}\right\}$ with the multiplicative noise term can be clearly seen. As Fig. 11 demonstrates, multiplicative noise term effects increase with the coherence $|\rho|$, whereas importance of the additive speckle noise decreases.

The previous analysis has shown that speckle noise, in all the covariance matrix terms, can be divided into two noise terms, whose contribution to the final speckle noise depends on the complex correlation coefficient $\rho$. In the following, a quantitative validation of the speckle noise model given by (42) is presented. This analysis is based on showing that real polarimetric data follow the theoretical mean and standard deviation values obtained in the first part of this paper. To perform this second analysis, data already presented in Fig. 9 are used. The speckle noise model validity is shown, without loss of generality, over the copolar term $S_{\mathrm{hh}} S_{\mathrm{vv}}^{*}$, as it presents the wider coherence range (for most of natural targets) of all the covariance matrix terms. These results can be extended to the rest of the covariance matrix terms. 

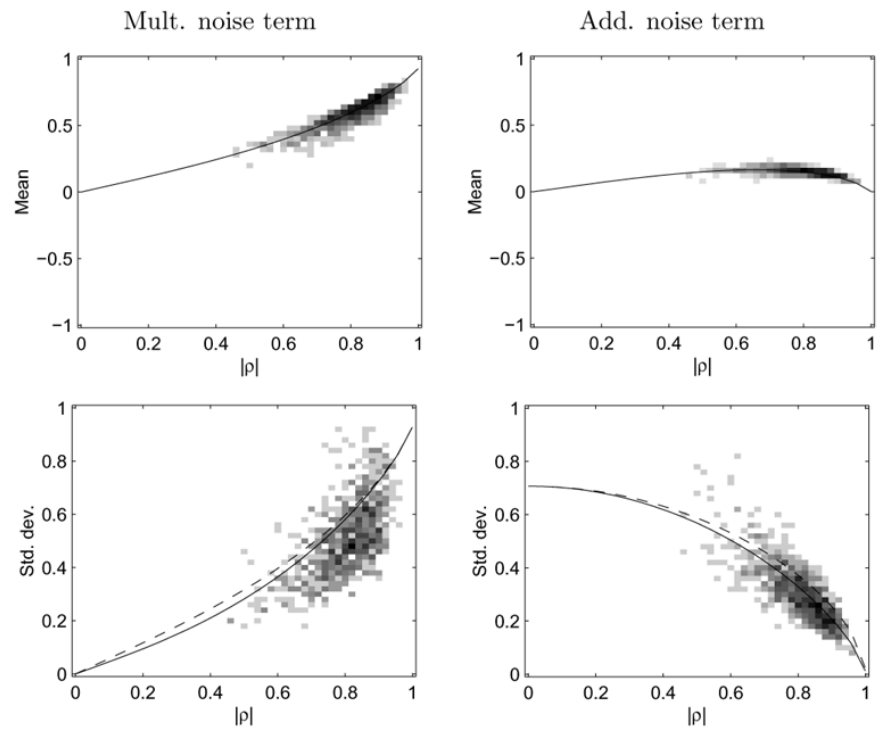

(a)

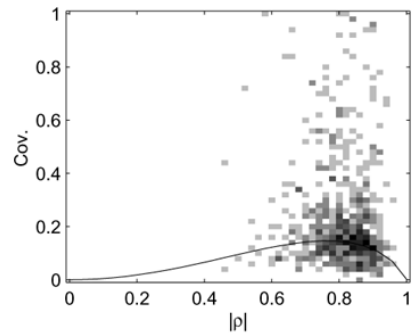

(b)

Fig. 12. Comparison between theoretical values (continuous and dashed lines) of mean and standard deviation for the different components of $\operatorname{Re}\left\{S_{\mathrm{hh}} S_{\mathrm{vv}}^{*}\right\}$ for the area with $\rho$ equal to $0.79 \exp (j 0.39)$ and values estimated from data. (a) Mean and standard deviations of the multiplicative and additive terms. (b) Covariance of the multiplicative and additive terms.

The validation process is based on analyzing two homogeneous areas of the dataset. The first area corresponds to an area characterized by a complex correlation coefficient equal to $0.79 \exp (j 0.39)$, where the phase is expressed in radians. The second region is characterized by a complex correlation coefficient with the value $0.36 \exp (-j 0.12)$, i.e., a low-coherence area. In each case, the mean and the standard deviation are calculated over $7 \times 7$ pixel nonoverlapped windows. In Figs. 12-14, solid lines represent the theoretical values of the statistical parameters, whereas dashed lines show the approximated values in the case of the standard deviation. As it can be observed in Figs. 12-14, the approximated values are very close to the actual ones, and they allow to obtain a linear speckle noise model.

Fig. 12 presents the analysis performed over the real part of the high-correlation area, i.e., $\operatorname{Re}\left\{S_{\mathrm{hh}} S_{\mathrm{vv}}^{*}\right\}$, whereas Fig. 13 gives the results for $\operatorname{Im}\left\{S_{\mathrm{hh}} S_{\mathrm{vv}}^{*}\right\}$. The graphics present density information coded by gray level: black for high densities, whereas white for low ones. For the sake of simplicity, data have been normalized by $\psi$, as this parameter acts as an scaling factor. At a first sight, the complete accordance between the data and the values given by the theory is obvious. This agreement is specially high for the mean values. Some important points have to be commented on for these data. As mentioned, the analyzed
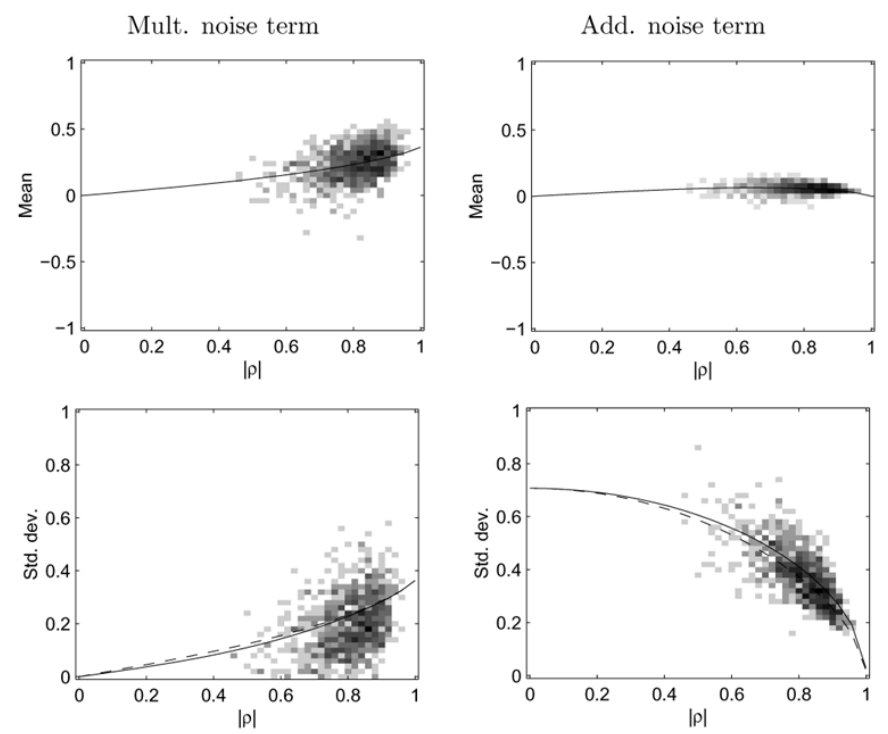

(a)

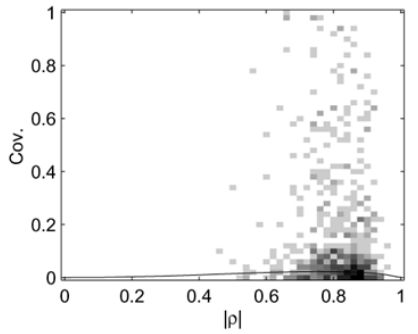

(b)

Fig. 13. Comparison between theoretical values (continuous and dashed lines) of mean and standard deviation for the different components of $\operatorname{Im}\left\{S_{\mathrm{hh}} S_{\mathrm{vv}}^{*}\right\}$ for the area with $\rho$ equal to $0.79 \exp (j 0.39)$. (a) Mean and standard deviations of the multiplicative and additive terms. (b) Covariance of the multiplicative and additive terms.

area has a high coherence. As it can be observed in Fig. 12, the multiplicative speckle noise term is more important than the additive speckle term, in standard deviation terms. But this situation changes for the imaginary part. In this case, it is possible to see that the additive speckle term is more important than the multiplicative one in terms of standard deviation. Therefore, as mentioned before, the speckle noise characteristics depend on the value of the complex correlation coefficient, as it determines the way in which the multiplicative and the additive noise terms are combined. Thus, it is also necessary to take into consideration the differences between the real and imaginary parts of speckle noise.

The agreement between theoretical and real values for the mean and standard deviation of the different terms of the proposed speckle noise model has been measured by a linear least squares regression analysis. The results concerning the high-coherence area are given in Table I. This table shows three parameters concerning the regression analysis. Two are $a_{0}$ and $a_{1}$, which refer to the regression line constant parameter and the slope respectively, whose values in case of a total agreement should be zero for $a_{0}$ and one for $a_{1}$. The third parameter is the coefficient of determination $r$, which measures the agreement between two quantities, quantifying it between zero and one. It can also be understood as a correlation measurement. 

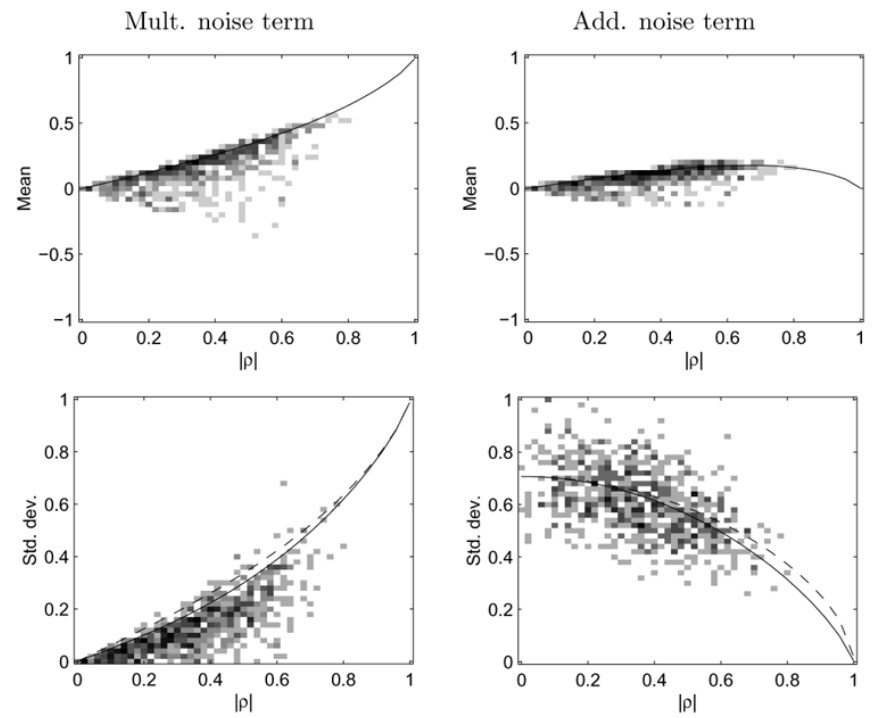

(a)

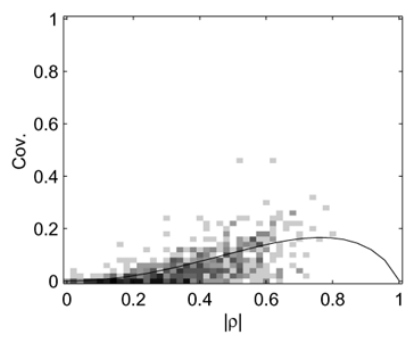

(b)

Fig. 14. Comparison between theoretical values (continuous and dashed lines) of mean and standard deviation for the different components of $\operatorname{Re}\left\{S_{\mathrm{hh}} S_{\mathrm{vv}}^{*}\right\}$ for the area with $\rho$ equal to $0.36 \exp (-j 0.12)$ and values estimated from data. (a) Mean and standard deviations of the multiplicative and additive terms. (b) Covariance of the multiplicative and additive terms.

TABLE I

LEAST SQUARES REgRESSION ANALYSIS RESULTS FOR THE HigH COHERENCE AREA $(0.79 \exp (j 0.39))$. (a) RESULtS FOR $\operatorname{Re}\left\{S_{\mathrm{hh}} S_{\mathrm{vv}}^{*}\right\}$. (b) RESULTS FOR $\operatorname{Im}\left\{S_{\mathrm{hh}} S_{\mathrm{vv}}^{*}\right\}$

\begin{tabular}{l|c|c|c|c}
\cline { 3 - 5 } \multicolumn{2}{c|}{} & \multicolumn{1}{c|}{$\mathrm{a}_{0}$} & $\mathrm{a}_{1}$ & $\mathrm{r}$ \\
\hline \multirow{2}{*}{ Mult. term } & Mean & 0 & 0.99 & 0.99 \\
\cline { 2 - 5 } & $\sigma$ & 0.03 & 0.84 & 0.47 \\
\hline \multirow{2}{*}{ Add. term } & Mean & 0.01 & 0.98 & 0.79 \\
\cline { 2 - 5 } & $\sigma$ & -0.01 & 0.98 & 0.69 \\
\hline
\end{tabular}

(a)

\begin{tabular}{l|c|r|c|c}
\cline { 3 - 5 } \multicolumn{2}{c|}{} & \multicolumn{1}{c|}{$\mathrm{a}_{0}$} & $\mathrm{a}_{1}$ & $\mathrm{r}$ \\
\hline \multirow{2}{*}{ Mult. term } & Mean & 0 & 0.99 & 0.99 \\
\cline { 2 - 5 } & $\sigma$ & 0 & 0.89 & 0.84 \\
\hline \multirow{2}{*}{ Add. term } & Mean & 0 & 1.03 & 0.97 \\
\cline { 2 - 5 } & $\sigma$ & -0.02 & 1.00 & 0.55 \\
\hline
\end{tabular}

(b)

From Table I, the total agreement between the theoretical and the measured values is clearly seen.

The noise model has also been analyzed over the low-coherence region. Fig. 14 presents the graphics at the statistical parameters concerning only $\operatorname{Re}\left\{S_{\mathrm{hh}} S_{\mathrm{vv}}^{*}\right\}$. As in the previous case, the complete agreement between theoretical and real values concerning the different statistical parameters can be observed. This agreement can also be seen from the least-square regression
TABLE II

LEAST SQUARES REGRESSION ANALYSIS RESULTS IN THE LOW COHERENCE AREA $(0.36 \exp (-j 0.12))$ FOR $\operatorname{Re}\left\{S_{\mathrm{hh}} S_{\mathrm{vv}}^{*}\right\}$

\begin{tabular}{c|c|c|c|c}
\cline { 3 - 5 } \multicolumn{2}{c|}{} & \multicolumn{1}{c|}{$\mathrm{a}_{0}$} & \multicolumn{1}{c|}{$\mathrm{a}_{1}$} & \multicolumn{1}{c}{$\mathrm{r}$} \\
\hline \multirow{2}{*}{ Mult. term } & Mean & 0 & 0.98 & 0.99 \\
\cline { 2 - 5 } & $\sigma$ & 0 & 0.90 & 0.88 \\
\hline \multirow{2}{*}{ Add. term } & Mean & 0 & 1.02 & 0.97 \\
\cline { 2 - 5 } & $\sigma$ & -0.01 & 0.98 & 0.26 \\
\hline
\end{tabular}

analysis done (cf. Table II). The imaginary part $\operatorname{Im}\left\{S_{\mathrm{hh}} S_{\mathrm{vv}}^{*}\right\}$ is not analyzed in this case, as it presents basically the same information as $\operatorname{Re}\left\{S_{\mathrm{hh}} S_{\mathrm{vv}}^{*}\right\}$.

All the covariance matrix terms, as presented along this paper, have the same statistical behavior. The difference between them lies in the fact that due to the scatterer properties, they will present different complex correlation coefficients. Hence, these results, obtained with $S_{\mathrm{hh}} S_{\mathrm{vv}}^{*}$, can be extended to the rest of the covariance matrix elements. The speckle noise model validity has been explicitly tested over the rest of the covariance matrix elements, obtaining in all the cases the same level of agreement.

\section{CONCLUSION}

A speckle noise model for the complete covariance matrix in PolSAR is proposed in this paper. This new speckle noise model allows to identify the noise characteristics for all the covariance matrix elements. As presented, this speckle noise model generalizes all the previous theory about speckle noise.

Small approximations have been necessary in order to obtain a linear speckle noise model. The speckle noise characteristics depend on the complex correlation coefficient $\rho$, causing the speckle noise nature to vary according to it. Two clear noise mechanisms have been identified. First of all, a multiplicative noise mechanism controlled by the real and imaginary parts of the complex correlation coefficient has been shown. This mechanism is dominant only when the real or imaginary parts of the complex correlation coefficient are close to one. The second mechanism has an additive nature, being dominant for low-coherence values. As a result, speckle noise for the off-diagonal covariance matrix elements is nonstationary, but also speckle characteristics vary between its real and imaginary parts.

The model has been validated with real L-band PolSAR data, obtaining a total agreement between the data and the developed theory.

\section{ACKNOWLEDGMENT}

The authors would like to express gratitude to the High Frequency and Radar Systems Institute (HR) from DLR for providing the Oberpfaffenhofen polarimetric dataset and in particular to K. P. Papathanassiou for his useful comments. Finally, the authors would like to thank the anonymous reviewers for their useful comments that contributed to improve the paper.

\section{REFERENCES}

[1] J. C. Curlander and R. N. McDonough, Synthetic Aperture Radar: Systems and Signal Processing. New York: Wiley, 1991.

[2] F. T. Ulaby and C. Elachi, Radar Polarimetry for Geoscience Applications. Norwood, MA: Artech House, 1990. 
[3] J. W. Goodman, "Some fundamental properties of speckle," J. Opt. Soc. Amer., vol. 66, no. 11, pp. 1145-1149, Nov. 1976.

[4] - Statistical Optics. New York: Wiley, 1984.

[5] J. S. Lee, "Speckle analysis and smoothing of synthetic aperture radar images," Comput. Graph. Image Process., vol. 17, pp. 24-32, 1981.

[6] A. Lopes, R. Touzi, and E. Nezry, "Adaptive speckle filters and scene hetereogeneity," IEEE Trans. Geosci. Remote Sensing, vol. 28, pp. 992-1000, Nov. 1990.

[7] C. Oliver and S. Quegan, Understanding Synthetic Aperture Radar Images. Norwood, MA: Artech House, 1998.

[8] J. S. Lee, M. R. Grunes, and G. de Grandi, "Polarimetric SAR speckle filtering and its impact for classification," IEEE Trans. Geosci. Remote Sensing, vol. 37, pp. 2363-2373, Sept. 1999.

[9] L. M. Novak and M. C. Burl, "Optimal speckle reduction polarimetric SAR imagery," IEEE Trans. Aerosp. Electron. Syst., vol. 26, pp. 293-305, Mar. 1990.

[10] A. Lopés and F. Séry, "Optimal speckle reduction for the product model in multilook polarimetric SAR imagery and the wishart distribution," IEEE Trans. Geosci. Remote Sensing, vol. 35, pp. 632-647, May 1997.

[11] J. S. Lee, M. R. Grunes, and S. A. Mango, "Speckle reduction in multipolarization, multifrequency SAR imagery," IEEE Trans. Geosci. Remote Sensing, vol. 29, pp. 535-544, July 1991.

[12] J. Schou and H. Skriver, "Restoration of polarimetric SAR images using simulated annealing," IEEE Trans. Geosci. Remote Sensing, vol. 39, pp. 2005-2016, Sept. 2001.

[13] S. R. Cloude and E. Pottier, "A review of target decomposition theorems in radar polarimetry," IEEE Trans. Geosci. Remote Sensing, vol. 34, pp. 498-518, Mar. 1996.

[14] R. M. A. Azzam and N. M. Bashara, Ellipsometry and Polarized Light. Amsterdam, The Netherlands: Elsevier, 1987.

[15] J. S. Lee, K. W. Hoppel, and S. A. Mango, "Intensity and phase statistics of multilook polarimetric and interferometric SAR imagery," IEEE Trans. Geosci. Remote Sensing, vol. 32, pp. 1017-1027, Sept. 1994.

[16] R. J. A. Tough, D. Blacknell, and S. Quegan, "A statistical description of polarimetric and interferometric synthetic aperture radar data," in Proc. R. Soc. London A, vol. 449, 1995, pp. 567-589.

[17] R. Touzi and A. Lopes, "The principle of speckle filtering in polarimetric SAR imagery," IEEE Trans. Geosci. Remote Sensing, vol. 32, pp. 1110-1114, Sept. 1994.

[18] N. R. Goodman, "Statistical analysis based on a certain multivariate complex gaussian distribution (an introduction)," Ann. Math. Statist., vol. 34, pp. 152-177, 1963.

[19] J. S. Lee, K. P. Papathanassiou, T. L. Ainsworth, M. R. Grunes, and A. Reigber, "A new technique for noise filtering of SAR interferometric phase images," IEEE Trans. Geosci. Remote Sensing, vol. 36, pp. 1456-1465, Sept. 1998.

[20] C. López-Martínez and X. Fábregas, "Modeling and reduction of SAR interferometric phase noise in the wavelet domain," IEEE Trans. Geosci. Remote Sensing, vol. 40, pp. 2553-2566, Dec. 2002.
[21] A. Papoulis, Probability, Random Variables and Stochastic Processes. New York: McGraw-Hill, 1984.

[22] A. Lopes, R. Mougin, A. Beaudoin, S. Goze, E. Nezry, R. Touzi, M. A. Karam, and A. K. Fung, "Phase difference statistics related to sensor and forest parameters," in Proc. IGARSS, 1992, pp. 779-781.

[23] K. Sarabandi, "Deviation of phase statistics from the mueller matrix," Radio Sci., vol. 27, no. 5, pp. 553-560, Sept. 1992.

[24] P. Graczyk, G. Letac, and H. Massam, "The complex wishart distribution and the symntetric group," Ann. Statist., vol. 31, no. 1, Feb. 2003.

[25] A. B. Kostinski and W. M. Boerner, "On foundations of radar polarimetry," IEEE Trans. Antennas Propagat., vol. AP-34, pp. 1395-1403, Dec. 1986.

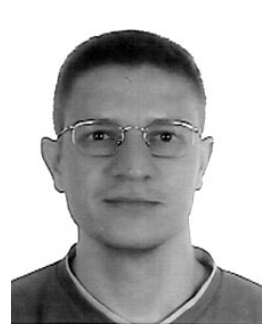

Carlos López-Martínez (S'97) received the M.S. degree in electrical engineering and the Ph.D. degree from the Technical University of Catalonia (UPC), Barcelona, Spain, in 1999 and 2003, respectively.

In 1999, he joined the Signal Theory and Communications Department, UPC, where he developed his Ph.D. thesis, which focused on multidimensional speckle noise modeling and reduction. From October 2000 until March 2002, he was with the High Frequency and Radar Systems Department (HR), German Aerospace Centre (DLR), Oberpfaffenhofen. Since June 2003, he has been with the Radar Polarimetry Remote Sensing Group of the Institute of Electronics and Telecommunications of Rennes, France. His research interests include SAR and multidimensional SAR, radar polarimetry, digital signal processing, and harmonic analysis.

Mr. López-Martínez received the Student Prize Paper Award at the EUSAR 2002 Conference, Cologne, Germany.

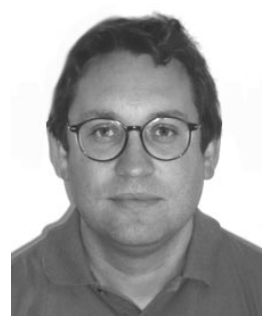

Xavier Fàbregas (M'90) received the B.S. degree in physics from Barcelona University, Barcelona, Spain, in 1988, and the Ph.D. degree in applied sciences from the Technical University of Catalonia (UPC), Barcelona, Spain, in 1995.

Since 1996, he has been Associate Professor with UPC. In 2001, he spent an eight-month sabbatical period at the High Frequency and Radar Systems Department, German Aerospace Center (DLR), Oberpfaffenhofen, Germany. His current research interests include polarimetric retrieval algorithms, polarimetric calibration, polarimetric SAR classification, and polarimetric weather radar analysis. 